Pure \& Appl. Chem., Vo1. 49, pp. 1507 - 1518. Pergamon Press, 1977. Printed in Great Britain. ANALYTICAL REQUIREMENTS IN EXPLORATION GEOCHEMISTRY

\author{
John S. Webb \& Michael Thompson
}

Applied Geochemistry Research Group, Imperial College, London SW7 2BP, England

Abstract - Geochemical surveys for mineral exploration are based on the systematic sampling and trace analysis of a wide range of naturally occurring materials, with a view to detecting anomalies related to concealed mineral deposits. A variety of cost-effective analytical systems have been specially devised for this purpose, mostly by more or less drastic modifications to conventional colorimetric and instrumental techniques. Success in this direction has only been achieved by analytical chemists and exploration geologists working together, with the common objective of helping to find mineral deposits as effectively and cheaply as possible. The ever-increasing complexities of the problems involved will continue to demand the closest possible interdisciplinary collaboration in the future.

\title{
INTRODUCTION
}

Exploration geochemistry encompasses any method of prospecting for mineral deposits based on the systematic sampling and analysis of naturally occurring materials. The commonest sampling media are rock, soil and stream sediment. Other media include vegetation, water, lake-bottom sediment, organic debris, soil gas and air. Whichever method is employed, the purpose is to determine spatial patterns in the distribution of the elements and specifically those abnormal patterns or geochemical anomalies related to the presence of concealed mineral deposits. Such anomalies are the result of natural processes resulting in dispersion of elements at the time of ore formation or during the course of weathering, soil formation and erosion.

The history of exploration geochemistry has largely been determined by three factors: (a) increasing demand for mineral resources coming at a time when most of the more obvious deposits had already been discovered; (b) advances in the concepts and understanding of geochemical processes bearing on dispersion of the elements; and (c) the development of rapid, low-cost analytical techniques for the determination of the elements in parts per million or even lower levels of concentration in solid, liquid and, more recently, gaseous media.

Inspired by the classical researches of A.E. Fersman, V.I. Vernadski and V.M. Goldschmidt, geochemical exploration in the modern sense was first practised in the USSR and Scandinavia in the 1930's, mostly based on soil and vegetation as the sampling media. In the late 1940's activity spread to the USA, Canada, Japan and thence to the UK and other countries in Europe, with concomitant studies in Africa, Australia, the Far East and many other parts of the world. During this period, attention was extended to include analysis of rocks, stream sediment and other sampling media mentioned above. Today, there are virtually no mineral fields in which geochemical surveys of one sort or another have not been made.

\section{GEOCHEMICAL SURVEYS}

In addition to classification according to the type of sample collected, geochemical surveys are also categorised according to their objective. Thus, primary regional geochemical reconnaissance surveys are aimed at evaluating many thousands of square kilometres with a view to elininating unfavourable ground and delineating potential mineralized districts as cheaply and as rapidly as possible. For this purpose, the technique is to sample at a chosen density of so many samples per unit area in order to delineate those areas in which to concentrate more detailed surveys aimed at detecting geochemical dispersion patterns related to individual mineral deposits. In detailed surveys, the samples will be taken at chosen intervals related to the expected extent of the dispersion patterns associated with the types of deposit sought. The objective at each successive stage is to reduce progressively the target size in the most cost-effective manner, prior to the final and most costly stage of any exploration programme, namely, the drilling required to locate and evaluate the mineralised deposit. In this context, it must be stressed that geochemical surveys are but one of the many aids available and that a well-directed exploration programme will include

Presented by Dr. C.H. James - University of Leicester. 
an appropriate integration of the geological, geophysical and geochemical techniques that exist for this purpose.

The basic concept of geochemical anomalies and phased geochemical exploration are illustrated by two simple examples in Figs. 1 and 2.

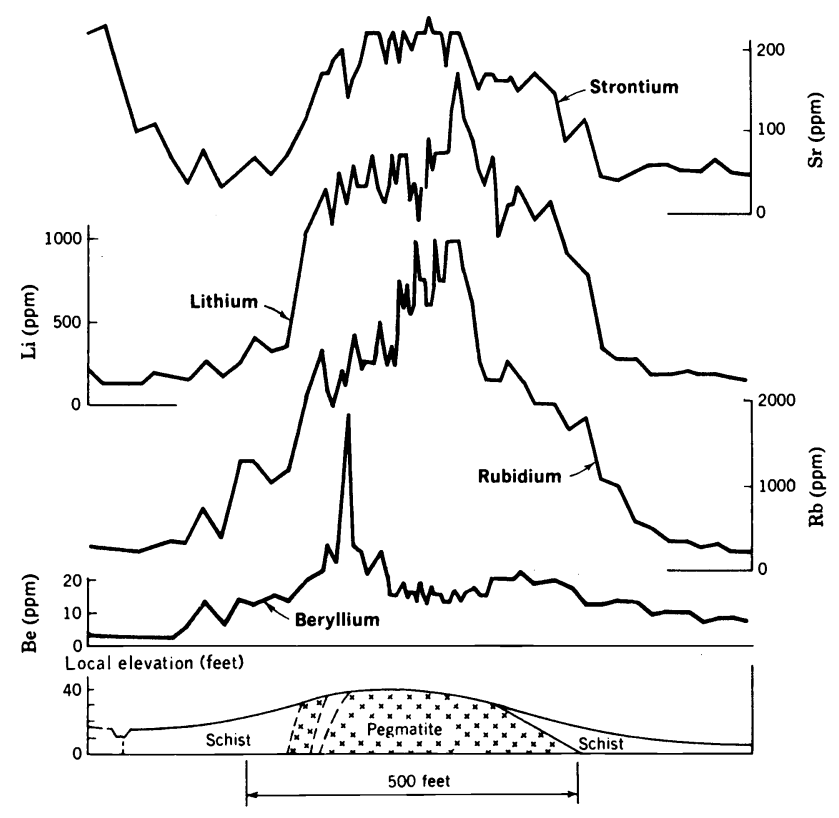

Fig. 1 Geochemical soil anomalies related to the underlying bedrock. Note beryllium anomaly indicating location of a beryl-rich zone in the pegmatite. (Hawkes \& Webb, 1962)

\section{Rock (1 ithogeochemical) surveys}

Most rock surveys depend on the development of patterns of abnormal metal concentrations preceding or during the period of mineralization.

At the reconnaissance level, rock surveys may be used to discriminate between potentially mineralized and barren geological formations. The trace metal content of the whole rock or selected mineral species is usually determined by the analysis of wide-spaced samples collected from surface outcrops. The average concentrations of some of the relevant trace elements in the more common rock-types are included in Table 1. The metal content of mineralized formations may be significantly higher than these normal background values for barren rocks. Similarly, the metal content of individual minerals may be higher than normal in mineralized rocks.

Detailed rock surveys have a number of applications. Metals may be dispersed into the wallrock enclosing a mineral deposit to form a geochemical aureole, thereby enlarging the exploration target. Wallrock aureoles may vary greatly in extent from a few metres or less up to several hundred metres or more, depending on local conditions. Traces of the ore metals and associated (pathfinder) elements may also be dispersed into the channel ways, followed by the mineralizing solutions. The resulting geochemical haloes or 'leakage' anomalies may then be detected by sampling the overlying rocks and pre-mineralization fracture systems.

So far, rock surveys have not been used to the same extent as soil or stream sediment surveys, mainly on account of the relative difficulty in obtaining representative samples, the variable distribution and area of outcrops and the need to collect relatively large samples which have to be finely ground prior to analysis. Nevertheless, there is a growing trend towards including rock sampling as an integral part of exploration programmes at some stage and, with more research advances, particularly in improved methods of interpreting the data, it is possible that this medium will find greater application in the future. 

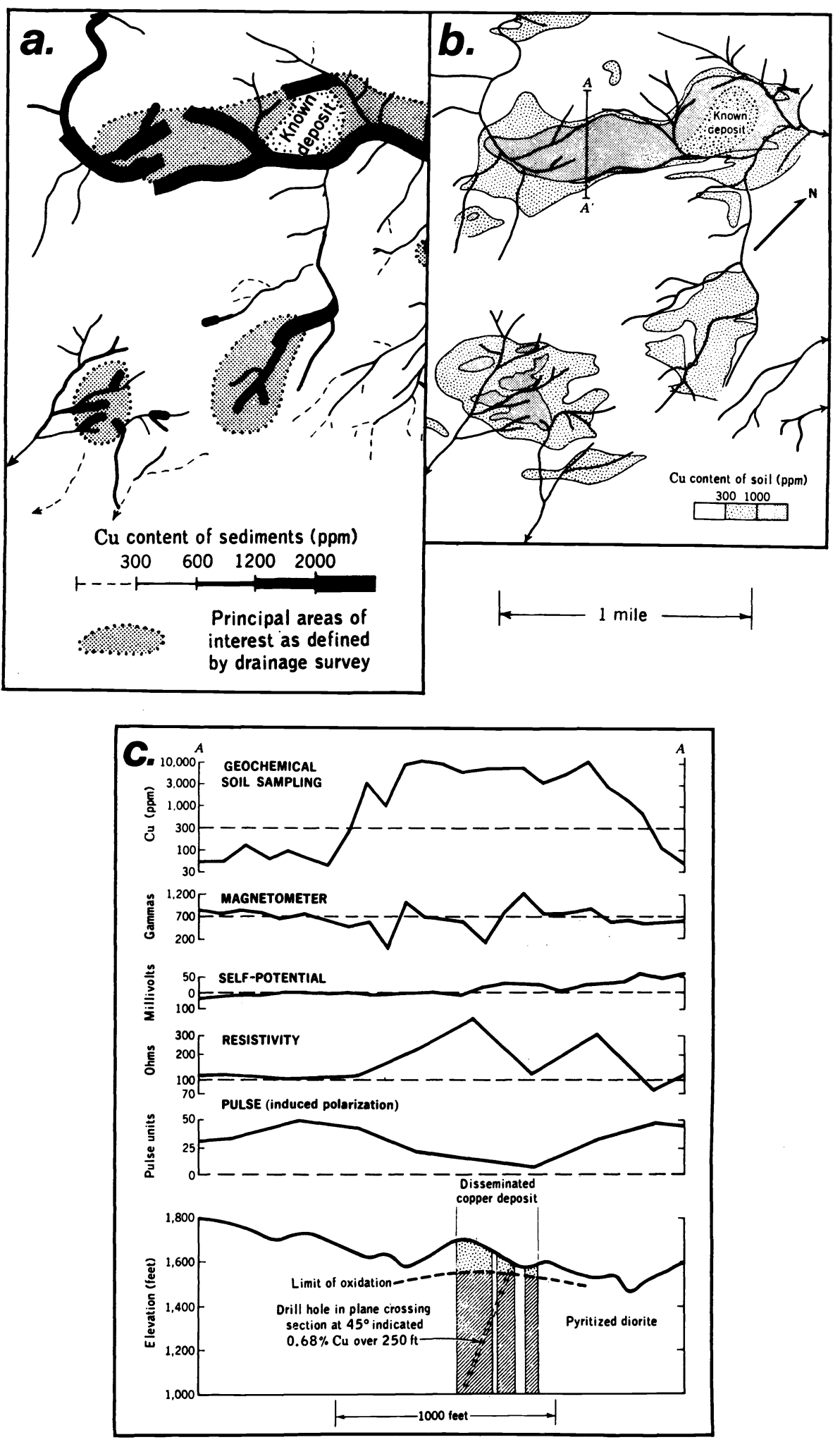

Fig.2 A typical case history illustrating phased exploration: (a) stream sediment reconnaissance to delineate possible areas of interest, (b) followup soil survey leading to (c) drilling of the target defined by multi-source data (Hawkes \& Webb, 1962) 
TABLE 1. Approximate orders of magnitude of background metal content for some of the media sampled in geochemical exploration surveys (considerable variation can be anticipated according to local conditions)

\begin{tabular}{|c|c|c|c|c|c|}
\hline Element & $\begin{array}{l}\text { Basic } \\
\text { igneous rocks } \\
(\mathrm{ppm})\end{array}$ & $\begin{array}{l}\text { Acid } \\
\text { igneous rocks } \\
(\mathrm{ppm})\end{array}$ & $\begin{array}{l}\text { Sedimentary } \\
\text { rocks } \\
(\mathrm{ppm})\end{array}$ & $\begin{array}{l}\text { Soils and } \\
\text { stream } \\
\text { sediment } \\
(\mathrm{ppm})\end{array}$ & $\begin{array}{l}\text { Fresh } \\
\text { water } \\
(\mu \mathrm{g} / 1)\end{array}$ \\
\hline$S$ & 2500 & 400 & 3000 & 850 & 5500 \\
\hline $\mathrm{Cr}$ & 1000 & 25 & 100 & 200 & 0.5 \\
\hline $\mathrm{Ni}$ & 700 & 8 & 10 & 40 & 0.02 \\
\hline$F$ & 200 & 800 & 150 & 200 & 50 \\
\hline $\mathrm{Ba}$ & 150 & 850 & 300 & 500 & 4 \\
\hline V & 150 & 40 & 50 & 100 & \\
\hline $\mathrm{Ca}$ & 100 & 5 & 5 & 8 & 0.03 \\
\hline $\mathrm{Cu}$ & 100 & 30 & 30 & 20 & 0.2 \\
\hline $\mathrm{Zn}$ & 90 & 60 & 20 & 50 & 10 \\
\hline $\mathrm{Pb}$ & 12 & 50 & 10 & 10 & 0.3 \\
\hline Sn & 6 & 45 & 10 & 10 & \\
\hline As & 2.5 & 1.5 & 4 & $<5$ & $<1$ \\
\hline$U$ & 0.5 & 3.5 & 2 & 1 & 0.05 \\
\hline $\mathrm{Ag}$ & 0.3 & 0.3 & 0.15 & 0.01 & 0.01 \\
\hline $\overrightarrow{c d}$ & 0.15 & 0.1 & 0.3 & 0.05 & \\
\hline $\mathrm{Hg}$ & 0.09 & 0.04 & 0.05 & 0.02 & 0.05 \\
\hline
\end{tabular}

Soil (pedogeochemical) surveys

Soil surveys have been used extensively and with considerable success under a wide range of conditions, particularly where the soils have developed on residual overburden. In these circumstances, soils over a sub-outcropping mineral deposit almost invariably contain greater quantities, albeit in minor or trace amounts, of the ore metals than do adjoining soils derived from barren materials. During the course of weathering and soil formation, the ore constituents are commonly dispersed laterally, resulting in soil anomalies considerably more extensive than the sub-outcrop of the deposit, especially in the nearsurface soil horizons.

Soil sampling may also be used in areas covered by glacial overburden. Near-surface soil sampling is effective providing the glacial debris is of local origin, although some 'smearing' of the anomaly in the direction of ice-movement is not uncommon. When the glacial debris is exotic, however, it may be necessary to sample near the bedrock surface in order to obtain satisfactory results.

While wide-spaced soil sampling has been used for regional surveys in some instances, soil surveys are mostly employed at the detailed level for following-up the results of reconnaissance surveys, screening geophysical anomalies or testing favourable geological structures and the possible extension of known deposits. For detailed surveys, samples are usually collected on a systematic grid pattern at intervals commensurable with the expected size of the anomalies and may range from some 10 metres to 100 metres or more. Sample collection and preparation are rapid and inexpensive; in many cases, it is only necessary to take a $100 \mathrm{~g}$ sample at $15-20 \mathrm{~cm}$ from the surface, oven-dried and sieved to minus 80 mesh prior to analysis.

Despite their simplicity and reliability, it is only proper to stress that soil surveys commonly yield a plethora of anomalies related to minor mineralization and to secondary enrichment processes, either indirectly related or even unconnected with mineralization. The problems of cost-effective interpretation can of ten be formidable.

\section{Stream sediment surveys}

Stream sediment represents nature's closest approximation to a composite sample of the clastic products of weathering upstream from the sampling site. For this reason, stream sediment surveys are commonly employed for reconnaissance purposes. The reconnaissance may be at two levels: (a) primary reconnaissance to delineate mineral districts by sampling at densities ranging from 1 sample per 2-250sq. km. and (b) secondary reconnaissance to detect stream sediment anomalies related to individual mineral deposits by sampling tributary streams at intervals of $500 \mathrm{~m}$ to $2 \mathrm{~km}$ or more, depending on the length of the dispersion train which can extend for as much as several kilometres downstream from the deposit.

The method is rapid and very cost-effective, providing there is an adequate surface drainage system and reasonable accessibility. Small $100 \mathrm{~g}$ composite samples of the active 
sediment are taken at each sample site, dried and sieved, usually to minus 80 mesh for analysis. Coarser or finer size-fractions may be desirable in some circumstances. Occasionally, organic material has been chosen as the sampling medium. Panned heavy mineral concentrates have also been used to good effect and in some areas, such as certain parts of the Canadian Shield, reconnaissance surveys have been based on sampling lake-bottom sediments.

As with other types of geochemical surveys, the principal problems are those of interpretation, which must be resolved as reliably as possible, having regard to the relatively high cost per unit area of detailed follow-up techniques, such as soil or rock sampling.

Other types of geochemical surveys

Rock, soil and stream sediment surveys account for over $90 \%$ of the samples collected for geochemical exploration programmes.

TABLE 2. Relative usage of different sampling media in North America in 1971 averaged from Levinson (1974) based on a survey conducted by the Association of Exploration Geochemists.

Sample media Percentage of total samples

\begin{tabular}{lr}
\hline Soils & 45.6 \\
Stream sediment & 23.2 \\
Rocks & 23.2 \\
Water & 4.3 \\
Vegetation & 2.6 \\
Air & 0.4 \\
Others & 0.6 \\
\hline
\end{tabular}

Nevertheless, other sampling media have important applications in certain circumstances, while yet others may find increasing use consequent upon the results of current and future research.

Water (hydrogeochemical) surveys With the exception of uranium, geochemical surveys based on water sampling (either surface or ground water) have not enjoyed general popularity. The principal reasons for this are: (a) susceptibility to fluctuation in metal content related to rainfall and seasonal variations; (b) the need to collect, store and stabilize large sample volumes; and (c) the problems of analysis in the parts per billion range, coupled with the problems of contamination.

Water may well receive increased attention, however, with the development of sensitive specialised analytical and sampling techniques and advances in the understanding of hydrogeochemical dispersion. Recent studies have also indicated the possibility of detecting significant anomalies by the analysis of snow.

Vegetation (biogeochemical and geobotanical) surveys Vegetation surveys rely on the fact that metals may be taken up by the roots and migrate to various parts of the plant or tree. Biogeochemical surveys based on analysis of the leaves, twigs or other plant organs may then show anomalous concentrations in those plants rooted in metal-rich soil or rock. The existence of such areas may also be detected by visual geobotanical surveys based on the preferential growth of certain metal-indicator species, diagnostic changes in the vegetation assemblage and in plant morphology.

The metal content of vegetation varies markedly, according to the factors governing the bioavailability of the metals in the root-zone, the plant species, its age, the organ sampled and the time of year. Furthermore, the metals in the vegetation are eventually returned to the soil as leaves fall and the plant dies, thereby building up over the years a relatively more homogeneous soil anomaly which is easier to sample and involves simpler preparation for analysis. For these reasons, biogeochemical surveys have not been used extensively in exploration, although they can be applicable under exceptional conditions, particularly in semi-arid areas of exotic overburden. There is far more scope for geobotanical surveys, justifying the training of more specialists in this promising field.

Vapour surveys With the exception of mercury and radon, vapour survey techniques are largely in the experimental stage of development and fall into two main categories based on sampling and analysis of soil gas and atmospheric air respectively. Mercury anomalies in soil air have been reported on a number of occasions over a variety of base-metal sulphide deposits, and radon has been used successfully in the search for uranium. A number of inorganic and organic sulphur gases, including volatile organometallic compounds, could well be developed during the oxidation of sulphide deposits, as well as the halogens (or their volatile compounds) which are associated with some types of mineralization. An increase in the $\mathrm{CO}_{2}: 0_{2}$ 
ratio in soil air has also been demonstrated over oxidising sulphides.

The detection of geochemical anomalies in the atmosphere is also receiving attention, with encouraging results. The analytical and interpretational problems are considerable, particularly in atmospheric surveys. Nevertheless, the inherent potential of utilising the mobility of gaseous and fine particulate emanations related to concealed mineralization is so attractive that there is little doubt that continued research will result in the development of commercially viable exploration techniques.

\section{ANALYTICAL REQUIREMENTS AND TECHNIQUES EMPLOYED}

Effective geochemical surveys comprise three distinct phases: (a) choice of the optimum systems for sampling and analysis based, whenever possible, on the results of a preliminary orientation survey in the vicinity of known mineralization; (b) efficient organization and conduct of the sampling and analytical routines; and (c) reliable interpretation of the results.

Each phase is equally important in ensuring a successful outcome but, in the context of this Symposium, we are primarily concerned with the analytical aspects and also, to a certain extent, with the sampling in so far as the field sampling error and expected contrast between anomalous and background values influence the analytical requirement.

Before reviewing briefly the more common analytical techniques employed in geochemical exploration programmes, it is necessary to outline the basic specification for any analytical system developed for this purpose:

\section{(a) Cost-effectiveness is the over-riding factor.}

(b) The elements determined should be the minimum required for adequate identification of the target sought and for screening significant and 'false' anomalies. There is, however, increasing recognition of the value of multi-element coverage for certain purposes, particularly primary multi-purpose regional geochemical mapping.

(c) Since the object is to determine patterns in the distribution of the diagnostic elements, precision is more important than absolute accuracy. Nevertheless, there is no point in increasing analytical costs in order to obtain a greater precision than is consistent with the field sampling error, which is usually, except in special circumstances, in the order of $\pm 25-50 \%$. Also in this connexion, the precision should be related to the expected significant contrast between anomalies and background values, which for most surveys commonly exceeds a factor of two-fold or more.

(d) The limit of detection (sensitivity) of the method should be such as to give positive readings in or near the background ranges likely to be encountered in the various sampling media; the values given in Table I refer to total metal content but, in practice, only partial determinations may be required in order to improve anomaly detection and/or to increase analytical throughput.

(e) Turn-round time must be as short as possible since very large numbers of samples are involved and the rate of collection is high. Hence the method must be rapid (in the order of at least 100+ analyses per man-day) and, if possible, carried out in the field, although for some techniques permanent laboratory facilities are desirable.

(f) The method should be capable of being operated reliably by trained but otherwise unqualified personnel, who should always, if possible, be operating under the supervision of a qualified analytical chemist working in close consultation with the exploration geochemist to ensure that the methods employed and the data obtained are kept in line with the requirements of the survey.

(g) In these circumstances, continuous monitoring of data quality is vital and $10-15 \%$ of analytical productivity is normally required for the regular insertion of appropriate control samples.

It is these requirements which have imposed upon geochemical exploration analysis its distinctive features, which arise from the need to combine stringent discipline with - to the conventional analytical chemist - startling short-cuts and simplifications. All labour intensive and time-consuming stages are cut to a minimum and methodology is reduced to the shortest and simplest possible set of instructions. While techniques involving low capital investment and running costs have obvious advantages, the very large analytical throughput may well justify the installation of quite costly, automatic or semi-automatic instrumentation.

Another factor in geochemical exploration analysis is that the major elements ( $>1$ per cent) comprising the bulk composition of the more common sampling media (soil, stream sediments and rocks) are $\mathrm{Si}, \mathrm{Al}, \mathrm{Na}, \mathrm{K}, \mathrm{Ca}, \mathrm{Mg}$ and $\mathrm{Fe}$. Matrix problems are, therefore, mostly limited to 
variations in the relative proportions of these elements, which can be considerable.

Historically, spectrographic methods were among the first used in geochemical exploration in the USSR and Scandinavia. In general, only the principal ore elements were determined at that time and the multi-element capacity of the instrument was largely unused. Application of geochemical methods in the Western countries stemmed primarily from the development of simple and rapid colorimetric methods by the US Geological Survey in 1946 and their subsequent extension at other centres between then and the mid-1950's. These methods, which depended on visual comparisons, were adapted so that they could be conducted by a non-chemist in the field, in some cases on the actual sample site. Soon after the appearance of the first commercial atomic absorption spectrophotometers, in the early 1960's, this technique rapidly proved its worth and became the most frequently used method in exploration work, a position which it still maintains. Recently, the value of multi-element determinations has become apparent and more precise spectrographic methods have been developed. This trend will, we believe, be continued by the increasing use of the induction-coupled plasma as a high precision source for emission spectrometry.

The present-day relative usage of the different analytical techniques are indicated in Table 3. The percentage applications given in this table are necessarily no more than approximate generalizations, but, with the exception of the USSR, where there is still a considerable dependence on emission spectrography, give what is probably a reasonable assessment of the present situation as a whole. Considered world-wide, the total number of samples collected per annum for exploration purposes is difficult to estimate, but is almost certainly well in excess of $10 \mathrm{million}$.

TABLE 3. Relative usage of analytical methods used in exploration geochemistry in North America in 1971 (averaged from Levinson (1974) based on a survey conducted by the Association of Exploration Geochemists)

$\begin{array}{lll}\text { Analytical } & \text { Percent of } & \text { Number of elements by } \\ \text { Method } & \text { total number } & \text { each method (max.) } \\ \text { of samples } & \end{array}$

\begin{tabular}{lrr} 
Atomic absorption & 69.4 & 40 \\
Colorimetry & 16.8 & 38 \\
Emission spectrography & 6.6 & 70 \\
X-ray fluorescence & 2.8 & 41 \\
Paper chromatography & 0.5 & 7 \\
Selective ion electrodes & 0.6 & 9 \\
Others & 3.3 & 35 \\
\hline
\end{tabular}

\section{Sample preparation}

As with all trace analysis, common sense precautions against contamination are mandatory at all stages, but for most practical purposes, these do not usually demand expensive refinements such as positive-pressure laboratories or laminar-flow fume cupboards. Sample preparation procedures must be geared to the most rapid and cheapest systems, to yield a sample sufficiently representative for the purposes of any particular survey programme. With certain exceptions, it is rarely necessary to go to the lengths of preparing a truly representative sample in order to acquire adequate data for exploration purposes. In each case, the most cost-effective system can best be worked out in consultation between the analyst and exploration geochemist, and preferably determined by the results of preliminary orientation studies carried out in the area to be surveyed.

For rock surveys, the sample procedure required is usually relatively conventional - samples of $100 \mathrm{~g}$ to $1 \mathrm{~kg}$ or more, depending on grainsize and the distribution of the element(s) sought, being reduced using the optimum combination of non-contaminating mechanical equipment for comminution and splitting to obtain a final sample of $5-30 \mathrm{~g}$ at minus 200 mesh. Soils and stream sediments, on the other hand, are usually collected in special wet-strength paper envelopes so that they can be oven- or air-dried without the need to transfer them to another container. The samples $(100-500 \mathrm{~g})$ are then simply disaggregated (not ground) in a pestle-and -mortar prior to sieving, using a plastic, nylon-mesh sieve commonly minus 80 mesh, though for some surveys coarser or finer fractions may be desirable. For most purposes, neither splitting nor grinding (except for the coarser fractions on occasion) are required prior to analysis.

Vegetation samples, usually consisting of twigs or leaves weighing some $50 \mathrm{~g}$, are dry-ashed in a furnace at $450-550^{\circ} \mathrm{C}$ or, if more volatile elements are sought, 'wet'-ashed by digestion, using a mixture of oxidising acids. Water samples (c.l litre) may be acidified with metalfree acid in the field to $\mathrm{pH} 2$ to avoid changes due to subsequent precipitation or adsorption onto the walls of the container. They may have been or require to be passed through a Milli- 
pore $(0.45 \mu)$ filter to remove particulates. Air sampling is still so experimental and varied that the problems of preparing air samples for analysis cannot usefully be discussed at this stage.

\section{Atomic absorption spectrophotometry}

Atomic absorption spectrophotometry (AAS) with conventional flame atomisation is the most widely used analytical technique in exploration geochemistry. This is due to several factors : (a) atomic absorption spectrophotometers are relatively cheap, robust and transportable instruments; (b) they are easy to operate by trained but unqualified staff; (c) throughput of samples is very high, as many as 2600 determinations per instrument in an eight-hour shift being quite feasible; (d) they are cheap to run; (e) easy to calibrate; ( $f$ ) several elements can be determined on a solution produced by a single attack on the sample; and ( $g$ ) AAS is well suited to a wide range of the trace elements of interest. These factors are common to any application of the technique, but it is the sample attack methodology which is most distinctive in analysis for exploration geochemistry.

The most widely used methods of digestion for rocks, soils and sediments employ the strong mineral acids and their mixtures, for example diluted $(1+1)$ hydrochloric acid, diluted $(1+1)$ nitric acid, aqua regia and nitric acid/perchloric acid (4+1). In most cases, these attacks do not extract the total metal content. Hydrofluoric acid is rarely employed in exploration work and fusion techniques give rise to problems associated with the large quantities of alkali metal salts in solution.

In typical procedures the samples $(0.25 \mathrm{~g}$ of 80 mesh (c. $200 \mu)$ material) are weighed in batches of 100 or more into borosilicate test tubes and digested for one hour with $1 \mathrm{ml}$ of the acid. After dilution to $10 \mathrm{ml}$ with water, the solid residue is left to settle. The solution is then nebulised directly from the test tube. Standards are prepared in the appropriate acid solution. Where a more rigorous attack is required (e.g. with nitric/perchloric acid mixture), the acid is slowly evaporated in a specially constructed air-bath. The dry residue is then leached with hydrochloric acid before dilution to $10 \mathrm{ml}$.

Some aspects of this procedure illustrate the special geochemical approach. Using an exact sample weight is not in itself time-consuming and eliminates calculations, as modern AAS instruments with linearisers can be set to give the concentration directly in ppm. Test tubes occupy less working surface than beakers and give a greater depth of solution for easy nebulisation. A single vessel is used for the whole procedure and there is no filtration. This saves a great deal of time which would be spent transferring solutions from one vessel to another. Also trace element losses onto vessel surfaces are minimised. While the attack is not total, it usually approaches $100 \%$ extraction if the appropriate acid is used, it is quite reproducible. The contribution to the total analytical variance from weighing and the attack is relatively small. Time is saved by avoiding exact adjustment of acid concentration and volume.

In contrast, there are several noteworthy sources of systematic and random errors. The unattacked residue, especially re-precipitated hydrous silica, can remove trace metals from solution by absorption. There are variations in the amount of acid lost during the heating stage, which result in variable final acid concentration and variable total volume, both of which contribute to variable sensitivity. There are variations in the amount of acid consumed by the samples and the amount of salts brought into solution, which can cause changes in surface tension and viscosity, affecting solution uptake rate and, therefore, sensitivity. Finally, there are errors due to variable amounts of major constituents which are also extracted from the samples and which may cause various types of interference.

of these errors, the random component is acceptably small. Within-batch precisions are often as low as $2-4 \%$ coefficient of variation (except, of course, at low concentrations approaching the detection limit). Overall precision (within-batch plus between-batch) is significantly worse (4-8\%), highlighting the great importance of minimising systematic changes between batches. Of the systematic errors, trace element loss through absorption is not a serious problem and, in a well-disciplined laboratory, neither is the opposite effect of sample contamination. By far the most pronounced error is due to interference from major constituents brought into solution and of these calcium produces the most serious effect, which is a 'back -ground' absorption. As a general rule in geochemical work, interferences which cause a change in the intercept of the calibration curve (translational changes) are more important than those which cause a change in slope (rotational changes). Concentrations measured are often at the bottom end of the curve, where translational changes have a more profound effect. Unfortunately, this interference is only partially eliminated by use of a continuous - source background correction system. However, as the effect is linear and additive to a reasonable approximation, it can of ten be compensated for by a separate calcium determination and occasionally by other methods.

Herbage samples are analysed by AAS after taking the ash into solution with acid or nebulising the solution directly in the case of 'wet' digestion. The level of heavy metals in natural waters is generally well below the working range of atomic absorption and consequently 
concentration methods have to be adopted. Solvent extraction with sodium diethyldithiocarbamate into chloroform is usually the preferred technique.

With the notable exception of mercury, flameless methods have had little impact on exploration work. Carbon furnace methods suffer from several disadvantages, namely (a) the calibration range is too narrow; (b) the method is much slower than flame methods; and (c) considerable skill and experience are required. The volatile hydride method (for $\mathrm{Ge}, \mathrm{Sn}, \mathrm{Pb}, \mathrm{As}, \mathrm{Sb}, \mathrm{Bi}, \mathrm{Se}, \mathrm{Te}$ ) looks promising, but is beset with interferences which still delay its application. In contrast, flameless methods for mercury have been established in exploration since the early 1960 's and have since proliferated into a number of variant techniques. Detection limits have reached as low as $5 \mathrm{ppb}$ and $5 \mathrm{ng}$ per cubic meter in soils and air respectively. The variety of the techniques evolved stems from the need to eliminate interferences from absorption by molecular species at the resonance line and the large number of ways by which this correction can be accomplished.

\section{Colorimetric methods}

Despite the ascendancy of AAS, rapid colorimetric methods are still widely used and, if anything, the philosophy of the exploration approach is even more marked. The methods are applied mainly to soil and sediment and are amenable to use in the laboratory or the field.

Sample preparation and attack are broadly similar to the methods described for AAS, but, in contrast, fusion, especially with potassium hydrogen sulphate, is a frequent substitute for treatment with a mineral acid. The resultant high concentrations of alkali metal have no undesirable effects in colorimetry and the solid reagent is more convenient to carry in the field. After the initial attack, an aliquot of the diluted acid is added to a fixed volume of a buffer-masking agent in a test tube and the metal of interest is extracted into a fixed volume of an organic solvent containing a suitable complexing reagent. The concentration of the metal is then estimated by visual comparison with extracts produced by the same method using a graduated series of standard solutions.

Reagents commonly used are dithizone for zinc and lead and $2,-2^{1}$ diquinolyl for copper. The practical limitations of visual acuity restrict the number of standard levels which can be differentiated to about ten, covering little more than one order of magnitude. Higher concentrations have to be brought within range by dilution. Rapid methods of this kind, suitable for in-field use by non-chemists, have been developed for a wide variety of metals and are capable of acceptable accuracy and precision.

Refinements of these methods combined with the use of spectrophotometers can often bring colorimetric methods to a standard comparable with AAS. At the present time, however, this usage is restricted to those elements for which AAS falls short of requirements, notably for $\mathrm{Mo}, \mathrm{W}, \mathrm{As}, \mathrm{Se}, \mathrm{Sn}$ and $\mathrm{Nb}$, and also $\mathrm{F}$. Modern spectrophotometers, with digital read-out and semiautomatic sample pick-up, allow determination rates comparable with AAS. In some cases, the method offers a useful alternative to AAS for elements where matrix interferences are difficult to eliminate.

Even more rapid are the relatively crude cold-extraction tests, where the sample, measured volumetrically, is simply shaken with a buffer solution and an organic solution of the complexing agent in a graduated test tube. Dithizone is the favoured reagent for 'heavy metals' $(\mathrm{Cu}, \mathrm{Pb}, \mathrm{Zn})$ because quantification is especially straightforward. The sample is 'titrated' by successive additions with shaking of the dithizone solution, until the organic layer takes up the greyish tint midway between the pure green of the reagent and the pink of the metal complex. The total volume of dithizone solution added being recorded as an approximate measure of the extractable metal content. These simple partial extraction methods were originally developed for use at the sample site, for which purpose their main function now is essentially restricted to the field follow-up of anomalies detected during the course of systematic surveys. The basic concept of determining partially extracted metal, however, has other practical applications as discussed below.

\section{Spectrographic methods}

In the methods which are currently employed, requiring a spectrograph of good dispersion, it is possible to select excitation conditions so that some 20-30 elements of interest can be simultaneously determined with adequate precision and detection limits. In a typical procedure, the minus 80 mesh fraction $(c .200 \mu \mathrm{m})$ of the soil or stream sediment is mechanically mixed with an equal weight of buffer containing lithium carbonate and carbon powder. The mixture is packed into the anode by repeatedly pressing the inverted electrode into a heap of the mixture, followed by smoothing of $f$ level with the crater rim. After development of the spectrographic plate, concentrations are assessed by visual comparison with the line intensities on a standard plate. Standards are made from mixtures of trace metal oxides in a synthetic matrix of composition approximating to that of the samples. Internal standards are not employed and precision is ensured only by the experience of the analyst packing the electrodes. Many elements can be determined with precision approaching $20 \%$ (coefficient of variation). Elements which are found predominantly in discrete mineral grains, however, such as tin, gold and zirconium, are much less satisfactory, because of the large sub-sampling 
variance inevitable with such small sample weights (about 15mg). Fine grinding of the sample with the buffer will usually improve the precision, although not greatly. Quoted precision estimates for these methods are liable to be misleading as they are invariably based on statistics valid for continuous variables.

The main drawback of photographic spectrography is the considerable time and skill involved in reading the plates. This task is removed in direct-reading spectrography, where the intensities of selected lines are measured by photomultipliers. In such instruments, the output of data is automatic and it is common for these instruments to be coupled directly to a dedicated computer which holds the calibration and correction data and outputs the results directly in concentration units. The sample rate can be as high as 200 or more samples per day. The precision of photoelectric measurement is inherently better than that of photographic plates, though the overall precision is still affected by the usual sources of variance from the direct current arc. Detection limits are usually slightly better.

The labour-reducing quality of direct-readers is offset by a number of drawbacks. Firstly, the selection of analytical lines is limited by engineering considerations, as well as spectrochemical requirements. Moreover, it is a difficult and expensive task to change or add to the original selection of lines. Secondly, the problems caused by background radiation are much more serious in direct-reader work. They can be alleviated, however, by a variety of procedures, but even after such correction it is the variation in background intensity from sample to sample which effectively determines the detection limit of the method, rather than the sensitivity of the atomic line. This background radiation is connected with the matrix composition of the sample and is especially prominent in calcareous samples. Arc-type interferences are considerably reduced by the use of alkali-loaded buffers, but there are still some residual effects and it is usual to match the matrix of the sample with a comparable synthetic matrix for the standards. Internal standardization is generally not effective in this type of work, probably because the samples are not finely ground with the buffer. A few important ore metals cannot be determined satisfactorily by these general methods. Among them are zinc (detection limit c.50 ppm), tungsten (c.50 ppm) and arsenic (c. $100 \mathrm{ppm})$.

The excitation of samples for atomic emission spectrometry by means of a high-frequency induction-coupled argon plasma is a technique which seems likely to replace the d.c. arc completely within a few years, despite the fact that in practical situations the performance does not come up to the levels shown feasible under ideal conditions. The sensitivity is very good and linear calibrations spanning four or more orders of magnitude in concentrations are reported for most elements. The stability of the plasma is excellent and relative precisions of $1-2 \%$ in intensity measurements are reported. Applications in exploration geochemistry have yet to be reported, but seem likely to prove considerable. Samples have to be brought into solution, but this has compensating advantages. In particular, variations between samples due to uptake rate can be compensated for by internal standardisation, in distinction to the (usually) single-channel atomic absorption.

\section{Other analytical methods}

X-ray fluorescence (XRF), although popular for the determination of major and minor elements of rocks and minerals in geochemical work, generally has been relatively little used in exploration geochemistry. Apart from the cost of appropriate instrumentation, the principal reasons for this lie in the detection limits, which are typically of the order of $100 \mathrm{ppm}$ and the time required for analysis. Both of these limitations can be over-come to a degree by appropriate refinements and automation, but result overall in relatively high unit costs in so far as commercial exploration surveys are concerned. The use of XRF in this latter context has, therefore, mostly been limited to those elements which cannot be determined so reliably by other more economic methods (e.g. Nb, Ta, Zr and some lanthanides) and for semiquantitative multi-element scanning (supplementing emission spectrography) in the preliminary orientation phase of exploration programmes and in applied research in this field. Portable non-dispersive XRF equipment has found limited use for in-field determination of a small range of ore-metals, notably $t$ in, but the limit of detection is too high for most exploration purposes.

Paper chromatography has been used to a rather surprising extent, considering the competitiveness of other procedures. The most common elements to be determined by paper chromatography in exploration geochemistry have been copper-cobalt-nickel and uranium-thorium on single sample extractions. The method has certain advantages for analysis in the field, but will almost certainly continue to decline in the future.

In contrast, the specific ion electrode which is now the most favoured method for fluorine will, no doubt, find increasing application in exploration geochemistry. Its future applications for other ions is less certain, unless significant developments occur. 
Fluorimetry is the most important method used in the determination of uranium at sub-ppm levels, both in waters and also in solid media after fusion with an appropriate flux.

Neutron activation analysis is not normally used in geochemical exploration. The technique has great analytical potential, but the high cost is a major limiting factor but, in certain circumstances, it can have value for analysing limited numbers of samples at the orientation survey phase and for confirmatory purposes on critical samples.

Radiometric techniques are rarely employed in the laboratory for exploration purposes, but a variety of instrumentation has been developed based on gamma-spectrometry, widely used in the search for uranium and thorium.

In the field of vapour geochemistry, various techniques and instrumentation have been developed for the detection of radon and mercury in soil gas; the former relies on detecting alphadecay products and the latter on atomic absorption. Vapour geochemical research and development is still experimental, but there is little doubt that gas chromatography and G.C. massspectrography will prove to be indispensible in the future.

As previously stated, air-borne geochemical surveys (apart from those which employ gammaspectrometry) are even more experimental, but it seems that the most likely analytical approaches will be the analysis of particulate matter by specialised multi-element spectrometric techniques.

There are a number of other methods, such as mass spectrometry (including isotope-ratio measurements), polarography and anodic stripping voltametry, which have been investigated for special purposes, but have not been widely used in exploration.

\section{Selective partial extraction}

According to their origin and mode of dispersion, trace metals can be chemically bonded in rocks, soil, stream sediment, water and air in a variety of ways. Extraction procedures which distinguish between trace elements in different molecular environments can be most useful in exploration surveys for the following purposes: (a) enhancing the contrast between anomalous and background values and increasing the extent of the anomaly by selectively extracting the metal(s) derived from a weathering deposit, (b) as an aid to interpretation by helping to differentiate between anomalies related to mineralization and ' false' anomalies due to secondary environmental factors, and (c) providing the basis for a simple coldextraction test that can be used at the sample site when following-up anomalies detected during the course of reconnaissance surveys, particularly those based on regional stream sediment sampling.

A number of partial extractants (usually followed by colorimetry or AAS for determining the metal extracted) have been used in this connexion; for example, (a) EDTA solution for organically chelated ions, (b) ammonium acetate or citrate for ions held at exchange sites,

(c) mild reducing agents (hydroquinone) for ions held in manganese(IV) oxide phases,

(d) stronger reducing agents (sodium dithionite) for ions held by iron(III) oxide phases, and

(e) mild mineral acid attack to extract trace metals from the lattices of clay minerals.

The chemical basis for these partial attacks is often quite difficult to justify. The methods always 'overlap' in the sense that ions are usually removed from more than one molecular situation, and there is rarely any direct way of testing whether the attack is actually doing what it was specifically designed to do. Moreover, sample drying and/or storage can have a marked effect on the strength with which the ions are held in the various molecular sub-strates.

At present, evaluation of the practical value of these methods can only be done empirically during the course of the preliminary orientation study carried out in the area of interest, prior to establishing the optimum techniques to be used in the subsequent exploration survey. Nevertheless, there is every reason to believe that, with increasing knowledge of the partition of the metals during the course of dispersion, it will be possible to devise a more logical basis for developing partial extraction procedures which could have a considerable impact in geochemical exploration practice.

\section{FUTURE TRENDS}

Attention has already been drawn to some specific areas in which valuable advances are being made in the field of exploration geochemistry, such as the continued development and application of plasma-excitation spectrophotometry and quadripole mass spectrometry, the current interest in soil gas and atmospheric surveys, and in methods for ion speciation by partial extraction and other procedures. In addition, there are a number of more generalized aspects where applied analytical chemistry can contribute materially to mineral exploration in the future.

First, there is the inevitable need to be able to detect increasingly subtle geochemical indications of concealed mineralization. This will require the development of cost-effective 
methods for determining trace metals with greater precision and, for some elements, at lower limits of detection. The result of so doing will be to increase the number of apparently anomalous geochemical patterns obtained during the course of a survey and, since the cost of following-up such patterns is far greater than the cost of detecting them, it follows that analytical advances in this direction must be accompanied by concomitant advances in reducing sampling error and in improving geochemical criteria for differentiating between significant and 'false' anomalies. On the analytical side, data quality control, including randomisation of samples prior to analysis, will become ever-more important. Geochemically, we will need to know far more about the factors affecting metal dispersion and there will be ever-increasing use of computerised data processing systems. The optimum 'blend' will vary from area to area and with the purpose of each survey. Achieving this goal will require the further development of specialised interdisciplinary expertise on all fronts.

Secondly, there is a general lack of fast, convenient methods with adequate sensitivity for a number of metals and non-metals which are already known to have significance in exploration, such as $\mathrm{As}, \mathrm{Se}, \mathrm{Te}, \mathrm{Br}, \mathrm{I}, \mathrm{Bi}$ and Re, for example. Allied to this is the growing requirement for fast, accurate multi-element analysis, coupled with low limits of detection. Developments in this field are not only required for multi-element geochemical mapping, but would be invaluable in exploration geochemistry research, as previously mentioned.

Thirdly, as al ready stated, in addition to cost-effectiveness, turn-round time is a vital factor in exploration. Consequently, there is the need for the further development of in situ field determination procedures, particularly the development of robust, reliable instrumentation capable of being operated by trained, but otherwise non-qualified analytical personnel.

Finally, the main theme of this paper. Effective geochemical exploration depends on the collection of the right sample, analysis by the right method, followed by reliable interpretation. All too frequently, the relationship between sampling and analysis is detached even within the same organisations - when the optimum combination can only be achieved by close collaboration and consultation between the geochemist and the analytical chemist, working together to solve the common problems on hand. Furthermore, the situation is dynamic on both fronts. The enormous strides currently being made in electronics with fundamental developments in instrumentation at competitive prices, are opening up the way to determining trace elements at very low concentrations that would have been entirely uneconomic in exploration only a few years ago. Geochemically, new problems in exploration are continually being encountered and research opens up new avenues which cannot be used commercially with currently available techniques. In short, the closest possible interdisciplinary collaboration - not only between the geochemist and the analytical chemist, but also the geologist, geophysicist, photogeologist (not forgetting the increasing availability of earth satellite imagery) and a host of related fields - is of paramount importance if the problems of maintaining resources of essential raw materials are to be overcome.

In a review of this nature covering such a broad field, a comprehensive bibliography is clearly impracticable. Consequently, two general texts $(1,2)$ are given for those analytical chemists wishing to obtain a general perspective of geochemical exploration, followed by references to comprehensive annotated bibliographies (3-8) which are cross-referenced both as to the element and analytical techniques employed.

\section{REFERENCES}

1. H.E. Hawkes and J.S. Webb, Geochemistry in Mineral Exploration, Harper and Row, New York (1962).

2. A.A. Levinson, Introduction to Exploration Chemistry, Applied Publishing Ltd. Calgary (1974).

3. J.W. Harbaugh, Geochemical Prospecting Abstracts through 1952, US Geo1. Surv. Bul1. 1000-A (1953).

4. J.E. Erikson, Geochemical Prospecting Abstracts July 1952-December 1954, US Geol. Surv. Bul1. 1000-G (1957).

5. E.L. Harkward, Geochemical Prospecting Abstracts January 1955-June 1957, US Geo1. Surv. Bul1. 1096-B (1961).

6. H.E. Hawkes et al, 1972 Supplement to Exploration Geochemistry Bibliography, Journal of Geochemical Exploration, 2, 41-45 (1973).

7. H.E. Hawkes et al, 1973 Supplement to Exploration Geochemistry Bibliography, Journal of Geochemical Exploration, 3, 89-128 (1974).

8. H.E. Hawkes et al, 1974 Supplement to Exploration Geochemistry Bibliography, Journal of Geochemical Exploration, 4, 271-314 (1975). 